\title{
Recognition memory and the recall of spatial location*
}

\author{
ARTHUR I. SCHULMAN \\ University of Virginia, Charlottesville, Virginia 22901
}

\begin{abstract}
It is argued that memory for events should depend on the ability to recall their attributes. Two experiments suggest that this is so for the attribute of spatial location. When words presented in spatial arrays were later re-presented in a joint test of recognition memory and location recall, it was found that the surer one's recognition, the greater the accuracy of location recall. Instructions to attend to a word's location resulted in poorer recognition memory and marginally poorer location recall than were obtained when location was "incidentally" learned. Finally, when words were presented in color as well as in space, recognition memory was best when both attributes were recalled, was better when only location was recalled than when only color was recalled, and was poorest when neither attribute was recalled.
\end{abstract}

When a distinctive visual context is associated with an event, memory for that event may be enhanced, as the classical method of loci reveals (Norman, 1969; Yates, 1966; Luria, 1968; Crovitz, 1970). But even in the absence of a special real or imagined context, spatial attributes may play a role in human memory.

People often remember where they saw things. We can even know where to look to find something of importance whose very character momentarily escapes us-an ability whose survival value may be greater than we realize. Such observations suggest that we may routinely record certain spatial information about events. Even when information about spatial context might seem irrelevant to an experience, its storage may enhance the memorability of that experience. The main purpose of the present research was to gather empirical support for this notion.

The experimental literature on memory for location is scanty. More than 70 years ago, however, Müller and Pilzecker (1900) discussed associations of "absolute place" in memorizing syllables. Jacobs (1907), working in Müller's laboratory, developed the idea of "external localization" of remembered words. Christal (1958), using a factor-analytic approach to isolate factors of visual memory, found that one of them was memory for "the position of objects in space." Underwood (1969) cites experiments that suggest the importance of spatial attributes in memory. More recent studies (Rothkopf, 1971; Zechmeister \& McKillip, 1971) show that memory for location on a printed page is better than chance allows and that recall of content is correlated with recall of location. Perhaps of greatest interest, however, is the work of Gamble and Wilson (1916). They presented 18 nonsense syllables in a three-row by six-column array. After the syllables were read as rhythmic pairs, the

\footnotetext{
*This paper incorporates data presented by the author at the 1971 meeting of the Eastern Psychological Association. Experiment I was carried out while the author was a staff member at the M.I.T. Lincoln Laboratory, a research laboratory operated with support from the U.S. Air Force.
}

odd-numbered syllables were re-presented; Ss had both to indicate a syllable's original location in the array and to name the syllable that had been paired with it. Location recall and associative recall were found to be highly correlated. Associative recall occurred $63 \%$ of the time when location was correctly recalled but only $12 \%$ of the time when it was not; the conditional percentages for location recall were $95 \%$ and $63 \%$, given (respectively) correct and incorrect associative recall.

In the two experiments reported here, words presented in a spatial array were later re-presented in a test of recognition memory. Under such conditions, it was predicted that the surer the recognition, the greater the likelihood that the attribute of spatial location would be recalled. Moreover, if it is true that the extraction of spatial information is a routine operation-if, in computer jargon, we are preprogrammed to record spatial attributes-then memory for location under conditions of "incidental" learning should be no worse than under conditions of "intentional" learning.

Experiment I was concerned with recognition memory and location recall, as well as the relation between them, when location information is acquired either incidentally or intentionally. In Experiment II, words were presented in color as well as in space, with color being learned intentionally and location incidentally. The focus here was on the relation between recognition memory and the recall of these stimulus attributes.

\section{EXPERIMENT I}

\section{Method}

Each of a sequence of 25 slides presented Ss-summer school undergraduates at Tufts University who were paid for their services-with words arrayed at each of the four cardinal points of the compass. Words were hand-printed directly on the slides. Each slide was displayed by a Kodak Carousel projector for $5 \mathrm{sec}$, including $0.75 \mathrm{sec}$ for change time. When the slide sequence terminated, $\mathrm{S}$ was tested on his ability to recognize the 
Table 1

Location Recall (L) as a Function of Confidence that an Old Word Was, in Fact, Old*

\begin{tabular}{lllll}
\hline & \multicolumn{2}{c}{ Group Inc } & \multicolumn{2}{c}{ Group Int } \\
\hline $\mathrm{P}(\mathrm{L} \mid 1)$ & .45 & $(676)$ & .40 & $(407)$ \\
$\mathrm{P}(\mathrm{L} \mid 2-4)$ & .28 & $(278)$ & .26 & $(362)$ \\
$\mathrm{P}(\mathrm{L} \mid 5-6)$ & .17 & $(230)$ & .24 & $(216)$ \\
\hline
\end{tabular}

*Parenthetical figures denote the number of responses on which a conditional probability is based. They fail to sum to 1200 (for Group Inc) and to 1000 (for Group Int) because of occasional omitted responses.

words just viewed and to recall their spatial location. All Ss in two experimental groups knew that a recognition test would follow the slide presentation. Group Int $(\mathrm{N}=10)$ also knew it would be tested for recall of a word's spatial location, while Group Inc $(\mathrm{N}=12)$ did not. Only the few minutes needed to instruct each group on the common memory task separated the slide presentation from the test of recognition memory and location recall.

The recognition test included all 100 "old" words interspersed at random among an equal number of "new" words. All words were nouns of two or three syllables and varied widely in their frequency of occurrence in the language. The median frequency was one per million words, according to the Thorndike-Lorge (1944) G count; some exceptionally unusual words were also included (Cieutat, 1963), as well as some familiar spondees and compound words not listed by Thorndike-Lorge. The recognition test was presented in booklet form. To the right of each word, four Xs were arranged in a diamond-shaped array, with an $X$ at each of the compass points at which a word might previously have appeared. To the right of this array were the numbers 1-6, representing a 6-point scale of (high-to-low) confidence that a word was "old." Working at his own pace, $S$ selected a compass point and a recognition confidence rating for each of the 200 words. Spatial recall was measured simply by the percentage of old words whose locations were given correctly. The use of confidence ratings permitted the determination of recognition memory operating characteristics (Egan, 1958), functions that reflect the difficulty of distinguishing old words from new. Normal use of a 6-point scale of confidence results in five coordinate pairs of "hit" and "false alarm" proportions to which an operating characteristic may be fitted (Green \& Swets, 1966).

\section{Results}

Operating characteristics were fitted by eye to each S's data. The functions obtained may fairly be described as linear on double probability paper, with slopes very close to 0.8 for nearly all Ss. For Group Inc, who did not know a location-recall test was coming, the mean value of $\mathrm{d}_{\mathrm{s}}$ was $1.32(\mathrm{~s}=0.34)$. For Group Int, who did know, $d_{s}=0.99(s=0.29) .{ }^{1}$ Ability to discriminate between old and new words thus favored Group Inc (Mann-Whitney U test, $\mathrm{p}<.05$ ), a perhaps counterintuitive result that will be discussed in a later section.

If Ss were only guessing, we would expect them to correctly recall a word's location $25 \%$ of the time. In fact, location recall averaged $35.3 \%(\mathrm{~s}=5.5)$ for Group Inc and $30.9 \%(s=5.6)$ for Group Int, both obviously above chance, with the difference again in favor of Group Inc (Mann-Whitney U test, $p<.10$ ). Even when we match subsets of seven Ss for $d_{s}$, we find better location recall-34.3\% vs $29.6 \%$-for the Ss from Group Inc.

Location recall may be examined as a function of S's confidence that a word was old. Table 1 presents these data, with Rating Categories 2.4 and 5.6 combined for the sake of reliability. For both groups, but especially for Group Inc, location recall was more likely when $S$ was confident in his recognition.

The relation between recognition confidence and location recall is more apparent if the analysis is confined to those Ss whose location recall was far above chance. (When $\mathrm{S}$ shows near-chance recall of location, we can hardly expect his probability of location recall to be ordered according to recognition confidence.) Twelve Ss-nine from Group Inc and three from Group Int-recalled 34 or more locations (the remaining Ss recalled 30 or fewer). For these Ss, $P(L \mid 1)=.48$, $\mathrm{P}(\mathrm{L} \mid 2-4)=.35$, and $\mathrm{P}(\mathrm{L} \mid 5.6)=.20$, where $\mathrm{L}$ denotes correct recall of location.

The relation between recognition memory and the recall of spatial location has an alternative expression as Fig. 1 shows. Here we see that the ability to discriminate between old and new words depends on whether or not a word's location is recalled. When location is not recalled, a single operating characteristic $\left(d_{s}=0.95\right)$ reasonably fits the data for both experimental groups. When location is recalled, recognition memory improves,

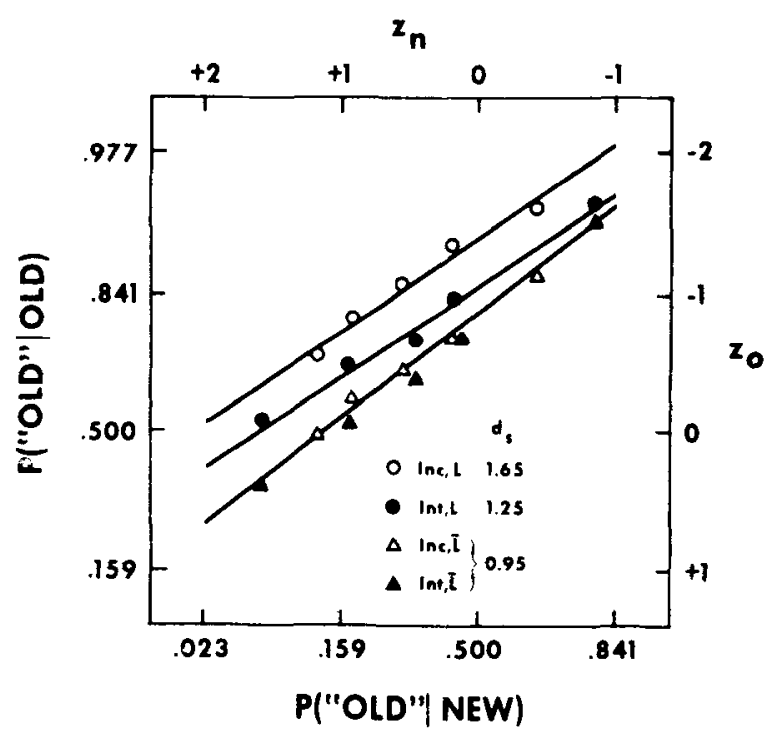

Fig. 1. Recognition memory for words whose spatial location was correctly (L) or incorrectly $(\overline{\mathrm{L}})$ recalled. For Groups Inc and Int, respectively, location had been learned incidentally and intentionally. The operating characteristics are based on pooled data; Ss contributed unequally to each. Ordinate values are based on 424 responses for Inc, $L ; 309$ for Int, $L ; 759$ for Inc, $\bar{L}$; and 676 for Int, $\overrightarrow{\mathrm{L}}$. Common abscissa values for Inc, $\mathrm{L}$ and $\operatorname{lnc}, \overrightarrow{\mathrm{L}}$ derive from 1,179 responses and for Int, $L$ and Int, $\bar{L}$ from 989 responses. (For Group Inc, there were 17 omitted responses to old words and 21 to new: for Group Int these figures were 15 and 11 , respectively.) 
but more for Group Inc than for Group Int $\left(\mathrm{d}_{\mathrm{s}}=1.65 \mathrm{vs}\right.$ $\mathrm{d}_{\mathrm{s}}=1.25$ ). Thus, Group Inc's superiority in recognition is wholly due to the fact that words whose location is recalled are better recognized after incidental than after intentional learning conditions.

\section{EXPERIMENT II}

\section{Method}

In this experiment, words were presented not only in a spatial array, but also in color. In addition to its spatial attribute, a word appeared in red, blue, green, or orange. Words were hand-printed in colored pencil directly on the slides. All four colors were represented on each of 24 slides, with a different color arrangement used for each slide. After the slide presentation, Ss were tested for word recognition and for recall of both word location and color. Like the uninstructed $S \mathrm{~s}$ of Experiment I, the 22 Ss here-undergraduates at the University of Virginia whose service was part of a course requirement-were not told they would be tested for recall of location. They were, however, forewarned about the recognition test (which here entailed a 4-point scale of confidence) and the test for color recall. The words to be recognized were two- or three-syllable nouns taken from Thorndike-Lorge (1944); their median G count was four per million. Ss were tested as a group. In all other respects as well, the procedure was the same as in Experiment I.

\section{Results}

Although location was learned "incidentally" and color "intentionally," location was recalled $35.0 \%$ of the time ( $\mathrm{s}=7.1$ ) to color's $29.1 \%(\mathrm{~s}=5.1)$. Of the $22 \mathrm{Ss}, 17$ recalled more locations than colors $(\mathrm{p}<.02$, sign test). The recall of color and location were not independent $\left(\chi^{2}=12.6, p<.001\right)$, but the extent of their dependence was not great. If $\mathrm{C}$ and $\mathrm{L}$ denote the recall of correct color and location, and if $\overline{\mathrm{C}}$ and $\overline{\mathrm{L}}$ denote incorrect recall of these attributes, then $\mathrm{P}(\mathrm{C} \mid \mathrm{L})=.34$ while $\mathrm{P}(\mathrm{C} \mid \overline{\mathrm{L}})=.27$ and $\mathrm{P}(\mathrm{L} \mid \mathrm{C})=.41$ while $\mathrm{P}(\mathrm{L} \mid \overline{\mathrm{C}})=$ 33 .

Table 2 indicates that, as in Experiment I, probability of location recall increases with recognition confidence. Those Ss with superior location recall-38 or more correct-again supply the clearest evidence for this, as Fig. 2 shows. Recognition confidence relates less clearly to color recall than to location recall, but with 15 of 22 Ss making 30 or fewer correct color judgments, there is little room for any order to be manifested.

The relation between recognition memory and the recall of stimulus attributes is summarized by the operating characteristics of Fig. 3. Recognition memory was best $\left(d_{s}=1.50\right)$ when both location and color were recalled, was better when only location was recalled $\left(d_{s}\right.$ $=1.10)$ than when only color was recalled $\left(d_{s}=0.84\right)$, and was poorest $\left(d_{s}=0.72\right)$ when neither attribute was recalled.

Recognition memory for a word did not depend on whether or not other words from the same slide were also recognized. To show this, only those confidence judgments involving a rating of "1" were examined. For
Table 2

Location Recall (L) and Color Recall (C) as a Function of Confidence that an Old Word Was, in Fact, Old*

\begin{tabular}{llll}
$\mathrm{P}(\mathrm{L} \mid 1)=.45$ & $(860)$ & $\mathrm{P}\left(\mathrm{C}^{\prime} \mid 1\right)=.35$ & $(860)$ \\
$\mathrm{P}(\mathrm{L} \mid 2)=.28$ & $(432)$ & $\mathrm{P}(\mathrm{C} \mid 2)=.21$ & $(432)$ \\
$\mathrm{P}(\mathrm{L} \mid 3)=.26$ & $(539)$ & $\mathrm{P}(\mathrm{C} \mid 3)=.25$ & $(539)$ \\
$\mathrm{P}(\mathrm{L} \mid 4)=.22$ & $(268)$ & $\mathrm{P}(\mathrm{C} \mid 4)=.27$ & $(268)$ \\
\hline
\end{tabular}

*Parenthetical figures denote the number of responses on which a conditional probability is based. They fail to sum to $22 \times 96=$ 2112 because of occasional omitted responses.

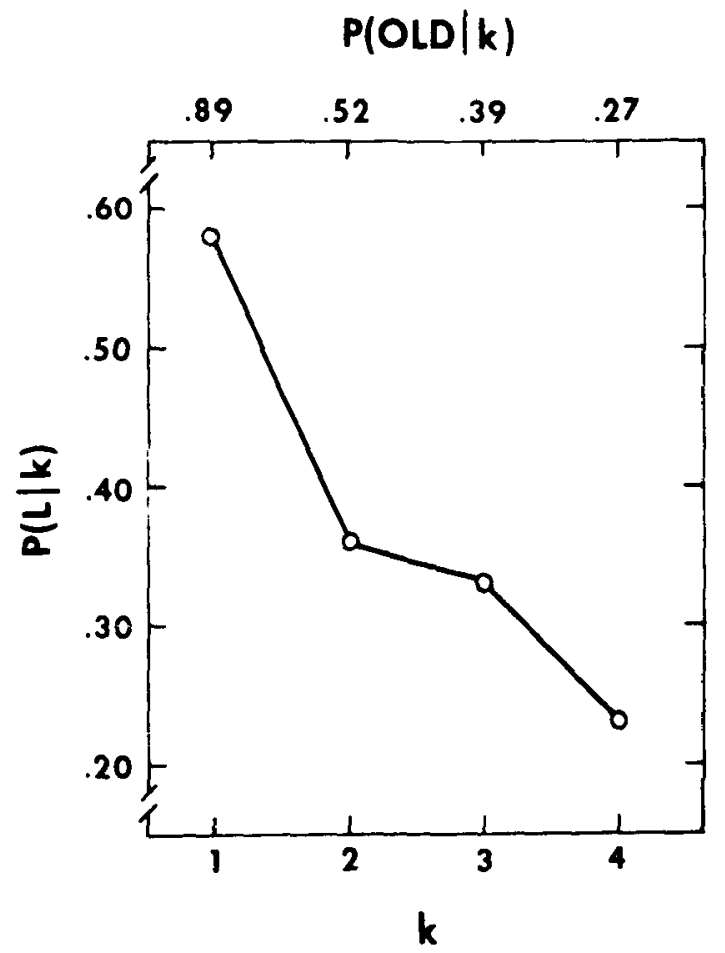

Fig. 2. Recall of a word's spatial location as a function of confidence in its recognition. Only the data from Ss with 38 or more correct location recalls (out of 96 possible) are shown. Ordinate values are based on 296, 109, 159, and 105 responses for $k=1,2,3$, and 4 , respectively. (These responses sum to 669 rather than $7 \times 96=672$ because of omissions by S.) The posterior probabilities that a word was in fact old, given the ratings as used by these Ss, are shown on the upper abscissa.

all Ss taken together, the number of slides for which 0 , $1,2,3$, and 4 words were correctly recognized was tabulated and compared with the expected number, given independence of processing. The calculated value of chi square was less than unity, thereby providing no evidence of intraslide word dependence.

\section{GENERAL DISCUSSION}

Experiments in recognition memory deal with the ability to remember that certain events occurred in a specifiable context. Usually the events are familiar and the context is recent. (With distant context, even recognition memory for pictures, normally excellent, is reduced to near-chance level: see Shepard, 1967.) An 


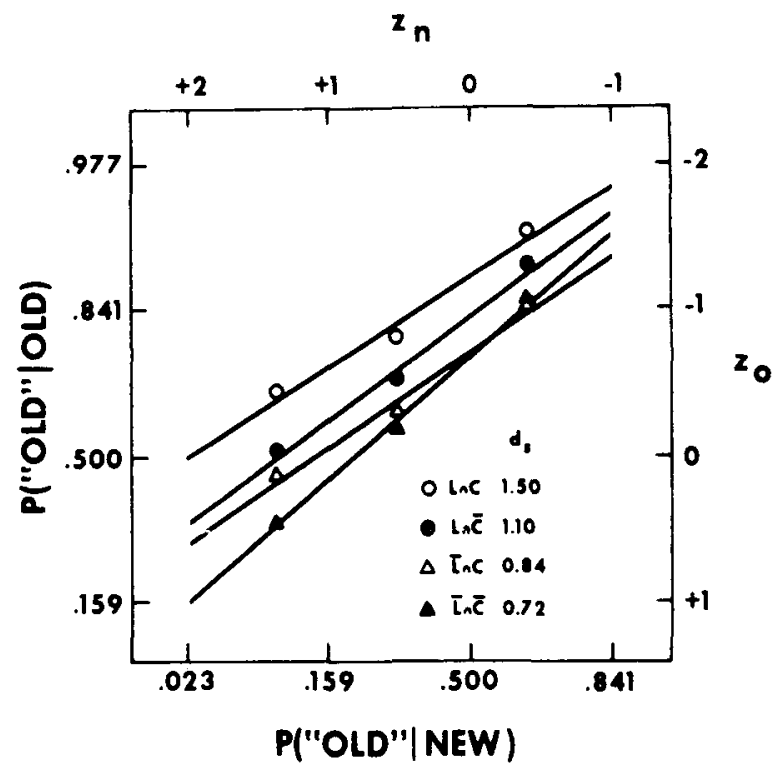

Fig. 3. Recognition memory and the recall of stimulus color (C) and location (L). The operating characteristics are based on pooled data: Ss contributed unequally to each. Ordinate values for these operating characteristics, from top to bottom, are based on $249,485,362$, and 1,003 responses. Their common abscissa values derive from 2,098 responses. (There were 13 omitted responses to old words, 14 to new).

event's context may be regarded as a set of attributes; Underwood (1969) goes so far as to propose that memory for an event consists of a collection of attributes.

We expect the restoration of the context in which an event was experienced to increase the chance that it will be remembered. Cued recall normally surpasses "free" recall, and cued recognition can surpass (cf. Winograd, Karchmer, \& Russell, 1971) uncued recognition. Such results make sense if the experience of an event involves its contextual encoding. What constitutes "context" is probably infinitely variable. Yet it may be useful to distinguish two kinds of attribute that an event may "have": those that can be considered apart from the event and those that are inseparable from it. Spatial location and color seem to be attributes of the latter sort.

I have tried to show that recognition memory and the recall of these attributes go hand in hand. While the results can support this view, one must acknowledge the logical possibility that recognition memory for an event may have nothing to do with the ability to recall its attributes. Were attribute recall merely to increase one's recognition confidence, without affecting $d_{s}$, the same relation between recall and recognition might emerge. How this relation might be explored so as to rule out an interpretation based on response bias is unfortunately far from obvious.
The results, then, must be cautiously evaluated. Certainly they are consistent with the notion that recognition memory for words varies directly with the ability to recall their arbitrarily assigned attributes of location and-to a lesser extent-of color. Location (i.e., position in space) seems to be an attribute that is routinely encoded, for its incidental recall is at least as good as its intentional. Indeed, instructions to learn location culminate in both poorer location recall and poorer recognition memory. We cannot be sure why this happens, but it seems reasonable to suppose that instructions to learn encourage verbal associations-shipwreck, north; firecracker, west-that reduce the effective time spent on each word and at the same time provide a poorer basis for location recall than the spatial information that would have been "automatically" recorded without those instructions. This supposition is of a piece with Eagle and Leiter's (1964) view that "intention to learn is crucial for learning only to the extent that it generates adequate learning operations." Recent findings of Paivio and Csapo (1973), showing that imagery benefits free recall even under conditions of incidental learning, are also consistent with the present argument.

The overall levels of location recall reported here are deceptively low. It is unreasonable to expect good attribute recall in the face of low overall levels of recognition memory. Besides, as Yntema and Mueser (1960) show, when a common set of four semantic attributes is shared by as few as eight variables, attribute recall is below $40 \%$ except when testing almost immediately follows input. In the present studies, with far more items with attributes to be recalled, with far greater average intervals between input and test, and with the use of a spatial (rather than a semantic) attribute, average location recall was still about $35 \%$. For the most confidently recognized words, this percentage jumps to about $45 \%$. (Unpublished work by the author has reproduced this level of location recall for sets of 40 words whose recognition memory could be assumed to be virtually without error.) A word's spatial location is clearly an attribute that is commonly encoded, frequently recalled, and intimately associated with the word's recognition.

Words and other events, of course, have defining attributes as well as those, like spatial location, that are peculiar to their current experience. Only a subset of these defining attributes may be "primed" when a familiar event is experienced, and not all of these may be equally important for later memory of the event. Unfortunately for theories of memory, it is not easy to see how research might disclose (a) which defining attributes are encoded and (b) the relation between recall of such attributes and memory for an event. 


\section{REFERENCES}

Cieutat, V. Association indices for 446 randomly selected English monosyllables, bisyllables, and trisyllables. Journal of Verbal Learning \& Verbal Behavior, 1963, 2, 176-185.

Christal, R. E. Factor analytic study of visual memory. Psychological Monographs, 1958, 72(13, Whole No. 466).

Crovitz, H. F. Galton's walk. New York: Harper \& Row, 1970.

Eagle, M., \& Leiter, E. Recall and recognition in intentional and incidental learning. Journal of Experimental Psychology, $1964,68,58-63$.

Egan, J. P. Recognition memory and the operating characteristic. Technical Note AFCRC-TN-58-51, 1958, Indiana University, Hearing and Communications Laboratory.

Gamble, E. A. McC., \& Wilson, L. A study of spatial associations in learning and in recall. Psychological Monographs, 1916, 22(4, Whole No. 96).

Green, D. M., \& Swets, J. A. Signal detection theory and psychophysics. New York: Wiley, 1966.

Jacobs, W. Lernen mit äusserer Lokalisation. Zeitschrift für Psychologie und Physiologie der Sinnesorgane, 1907, 45. 43-77.

Luria, A. R. The mind of a mnemonist. New York: Basic Books, 1968.

Müller, G. E., \& Pilzecker, A. Experimentelle Beiträge zur Lehre vom Gedächtnis. Zeitschrift für Psychologie und Physiologie der Sinnesorgane, Ergänzungsbund, 1900, 1, 1-288.

Norman, D. A. Memory and attention. New York: Wiley, 1969.

Paivio, A., \& Csapo, K. Picture superiority in free recall: Imagery or dual coding? Cognitive Psychology, 1973, in press.
Rothkopf, E. Z. Incidental memory for location of information in text. Journal of Verbal Learning \& Verbal Behavior, 1971, $10,608-613$

Shepard, R. N. Recognition memory for words. sentences, and pictures. Journal of Verbal Learning \& Verbal Behavior, 1967, 6. 156-163.

Thorndike, E. L., \& Lorge. I. The teacher's word book of 30,000 words. New York: Bureau of Publications, Teachers College, Columbia University, 1944.

Underwood. B. J. Attributes of memory. Psychological Review, $1969,76,559.573$.

Winograd, E., Karchmer, M. A., \& Russell, I. S. Role of encoding unitization in cued recognition memory. Journal of Verbal Learning \& Verbal Behavior, 1971, 10, 199.206.

Yates, F. A. The art of memory. Chicago: University of Chicago Press, 1966.

Yntema, D. B., \& Mueser, G. E. Remembering the present states of a number of variables. Journal of Experimental Psychology, $1960,60,18-22$.

Zechmeister, E. B., \& McKillip, J. Memory for place on a page. Paper presented at the 1971 meeting of the Midwestern Psychological Association.

\section{NOTE}

1. The index $d_{s}$ is defined as the difference between the $z$-score coordinates of the point at which the operating characteristic intersects the negative diagonal.

(Accepted for publication February 12, 1973.) 